Chapter 4

\title{
Multi-User Interference Suppression by Using Frequency Domain Adaptive Antenna Array
}

\author{
Wei Peng and Fumiyuki Adachi \\ Additional information is available at the end of the chapter \\ http://dx.doi.org/10.5772/57132
}

\section{Introduction}

The target data rate for the next generation wireless communication network will be around 1Gbps. To realize such a high data rate transmission, broadband transmission has been used in the current wireless communication network and it is also going to be employed by the next generation network. Due to the multi-path fading with large delay spread, broadband wireless channel is characterized by severe frequency selectivity [1]. As a result, it is necessary to suppress the inter-symbol interference (ISI) at the receiver. The ISI can be suppressed by time domain equalization techniques such as maximum likelihood sequence estimation (MLSE) [2]. However, when the data rate increases, the number of resolvable propagation paths increases as well and hence, the complexity of MLSE grows exponentially to the number of paths. Fortunately, the ISI problem can be solved by introducing frequency domain equalization (FDE) [3] at the receiver. It is well known that the frequency selectivity problem can be solved by the use of multi-carrier transmission technique such as orthogonal frequency division multiple access (OFDMA) [4] for the downlink (from base station (BS) to mobile users) transmission. However, the multicarrier transceivers are suffering from high peak-to-average power ratio (PAPR) problem which can lead to severe performance degradation. To solve the high PAPR problem, conventional single-carrier (SC) transmission, again, attracted much interest. Recently, the combination of SC-FDE and frequency division multiple access (called SC-FDMA) [5] has been considered as a more suitable solution for the uplink (from mobile users to BS) transmission. On the other hand, in order to save the bandwidth usage, the same carrier frequency/frequencies may be reused by neighboring cells to increase the bandwidth efficiency. As a result, co-channel interference (CCI) [6] becomes the dominant performance limitation instead of the thermal noise. In addition, multi-user interference (MUI) 
occurs when multiple users transmit simultaneously within the same cell (the MUI and CCI together is called multi-access interference (MAI)). Therefore, interference cancellation is necessary in uplink transmissions.

Recently, distributed antenna network (DAN) [7] has been proposed to solve the transmit power problem in broadband signal transmissions. As the data rate increases, impractically large transmit power will be required to realize the high data rate if cell coverage is kept unchanged. Otherwise, the cell coverage has to be reduced if the transmit power is kept unchanged. DAN was proposed as a solution to increase the cell coverage while maintaining the low transmit power. In the DAN, a number of antennas are distributed in each cell and those antennas are connected with the DAN central processor (which is similar to the BS in conventional cellular system) through optical cables. A mobile user can communicate with its' nearby located antennas even when it is at the cell edge. Therefore, the transmit power in DAN can be kept low while the coverage of the cell can be greatly increased.

In the previous studies [8, 9], a SC frequency domain adaptive antenna array (SCFDAAA) for the uplink transmission has been proposed and it has been shown that the SC-FDAAA can effectively suppress MAI in a severely frequency selective fading channel. In this article, we will present the performance of DAN SC-FDAAA and compare SCFDAAA in DAN and in conventional cellular system with centralized antennas at the BS (referred to as CAN system hereafter).

The rest of the article is organized as follows. The system model is given in Section II. SCFDAAA for DAN and CAN will be described in Section III. The post SC-FDAAA signal to interference plus noise (SINR) will be given in Section IV. The performance of SCFDAAA will be shown in Section V, both bit error rate (BER) distribution and the system capacity will be presented. Finally, the article will be concluded by Section VI.

\section{System model}

In cellular system, the same carrier frequency/frequencies will be reused in neighboring cells to effectively utilize the limited spectrum. Cellular structures with frequency reuse factors (FRFs) of 1, 3, 4 and 7 are shown in Fig. 1 as examples of frequency reuse. And FRF=1, 3, 4, 7, 9 and 12 will be considered in this study. The commonly used first layer CCI model is used here, i.e., only the CCI from the first layer neighboring cells will be considered and the number of CCI cells will be $B=6$. As stated in the Introduction, we are going to use both CAN and DAN in each cell. CAN and DAN structures are shown in Fig. 2. There are totally $N_{r}$ centralized / distributed antennas. In the DAN system, those antennas are connected to the DAN central processor by optical fibers. The received signals will be transmitted to the DAN central processor and in order to lower the cost, signal processing will be carried out by the DAN central processor. 


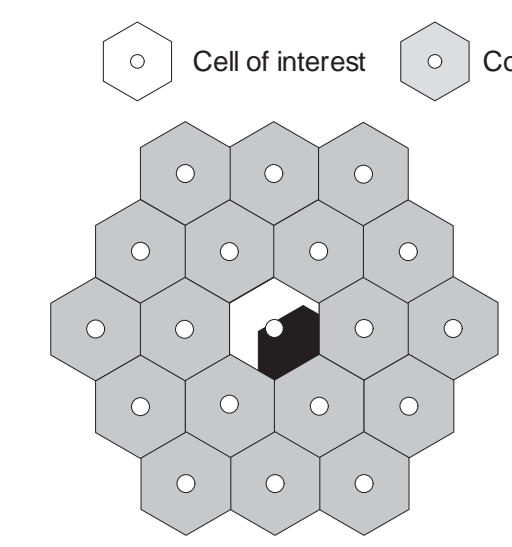

a) $\mathrm{FRF}=1$

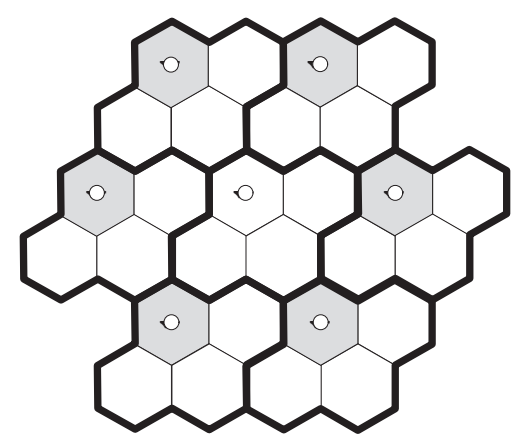

c) $\mathrm{FRF}=4$
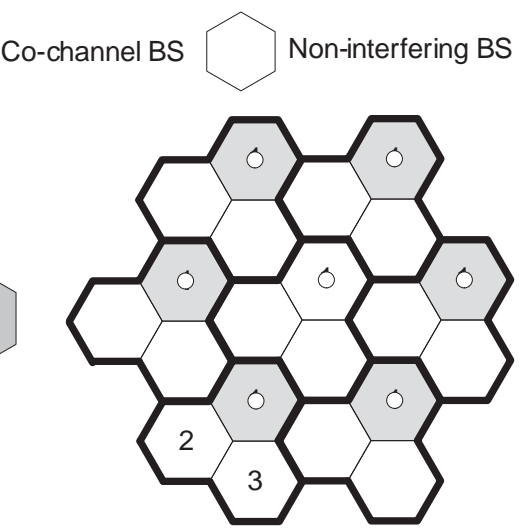

b) $\mathrm{FRF}=3$

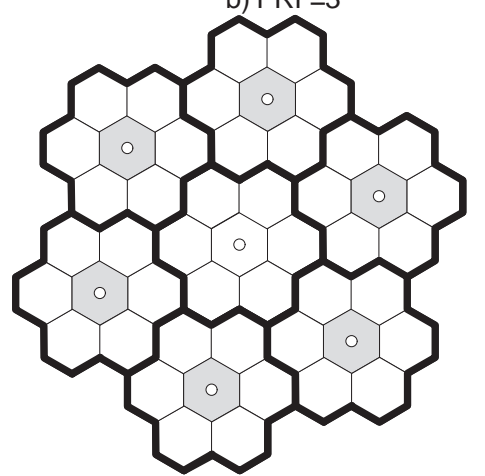

d) $\mathrm{FRF}=7$

Figure 1. Frequency reuse in cellular system.

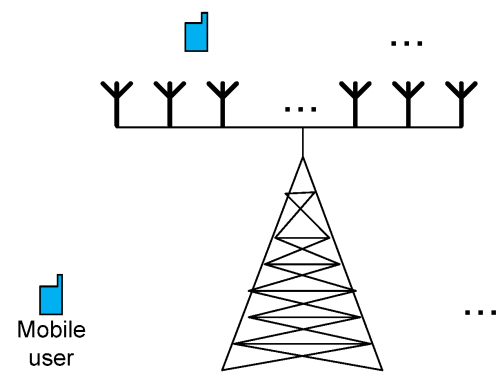

(a) $\mathrm{CAN}$

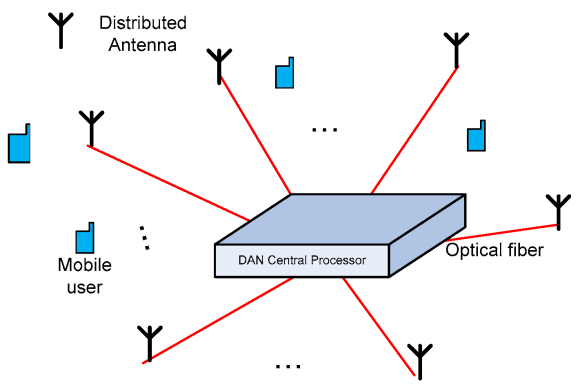

(b) DAN

Figure 2. CAN system and DAN system. 
It is assumed that there are $U$ users within each cell and each user is equipped with one omni antenna. A block fading channel between each user and each antenna is assumed, i.e., the channel remains unchanged during the transmission period of a block. In this article, the symbol-spaced discrete time representation of the signal is used.

Assuming an $L$ - path channel, the impulse response of the channel between the $u^{\text {th }}$ user and the $m^{\text {th }}$ antenna can be expressed as

$$
h_{u, m}(\tau)=\sum_{l=0}^{L-1} h_{u, m, l} \delta\left(\tau-\tau_{l}\right)
$$

where $h_{u, m, l}$ and $\tau_{l}$ are the path gain and time delay of the $l^{\text {th }}$ path, respectively. $h_{u, m, l}$ follows complex Gaussian distribution and satisfies $\sum_{l=0}^{L-1} E\left\{\left|h_{u, m, l}\right|{ }^{2}\right\}=1$, where $E\{\cdot\}$ represents the expectation. It is assumed that the time delay is a multiple integer of the symbol duration and $\tau_{l}=l$ is used. The cyclic-prefixed (CP) block signal transmission is used to make the received symbol block to be a circular convolution of the transmitted symbol block and the channel impulse response as well as to avoid inter block interference (IBI). It is also assumed that the $\mathrm{CP}$ is longer than the maximum path delay of the signal. In the following, we omit the insertion and removal of the $\mathrm{CP}$ for the simplicity.

The baseband equivalent received signal block $\left\{r_{m}(t) ; t=0 \sim N_{c}\right\}$ of $N_{c}$ symbols at the $m^{\text {th }}$ antenna is given by

$$
\begin{aligned}
r_{m}(t)= & \sqrt{P_{0} \delta_{0, m}^{-\alpha}} \sum_{l=0}^{L-1} h_{0, m, l} s_{0}(t-l)+\sum_{u=1}^{U-1} \sqrt{P_{u} \delta_{u, m}^{-\alpha}} \sum_{l=0}^{L-1} h_{u, m, l} s_{u}(t-l) \\
& +\sum_{i=1}^{B} \sum_{u_{i}=0}^{U_{i}-1} \sqrt{P_{i, u_{i}} \delta_{i, u_{i}, m}^{-\alpha}} \sum_{l=0}^{L-1} h_{u_{i}, m, l} s_{u_{i}}(t-l)+n_{m}(t),
\end{aligned}
$$

where $s_{u}(t)$ and $P_{u}$ are respectively the transmit signal and transmit signal power of the $u^{\text {th }}$ user $(u=0 \sim U-1) ; s_{u_{i}}$ and $P_{i, u_{i}}$ are respectively the transmit signal and transmit signal power of the $u_{i}^{\text {th }}$ user in the $i^{\text {th }}$ co-channel cell; $\delta_{0, m}$ represents the distance between the desired user and the $m^{\text {th }}$ antenna; $\delta_{i, m}$ represents the distance between the $i^{\text {th }}$ interfering user and the $m^{\text {th }}$ antenna; $\delta_{i, u_{i}, m}$ and $h_{u_{i}, m, l}$ are respectively the distance and channel gain between the CCI user and the $m^{\text {th }}$ antenna; $\alpha$ represents the path loss exponent in $\mathrm{dB}$; and $n_{m}(t)$ is the additive white Gaussian noise (AWGN). To simplify the analysis, no shadowing loss is assumed.

Let the transmit signal from the $u=0^{\text {th }}$ user be the desired signal and the transmit signals from the other users be the interfering signals. The frequency domain representation of (2) is given by 


$$
R_{m}(k)=H_{0, m}(k) S_{0}(k)+\sum_{u=1}^{U-1} H_{u, m}(k) S_{u}(k)+\sum_{i=1}^{B} \sum_{u_{i}=0}^{U_{i}-1} H_{u_{i}, m}(k) S_{i, u_{i}}(k)+N_{m}(k),
$$

where

$$
\left\{\begin{array}{l}
S_{u}(k)=\frac{1}{\sqrt{N_{c}}} \sqrt{P_{u} \delta_{u, m}^{-\alpha}} \sum_{t=0}^{N_{c}-1} s_{u}(t) \exp \left(-j 2 \pi k \frac{t}{N_{c}}\right) \\
S_{i, u_{i}}(k)=\frac{1}{\sqrt{N_{c}}} \sqrt{P_{u_{i}} \delta_{i, u_{i}, m}^{-\alpha}} \sum_{t=0}^{N_{c}-1} s_{i, u_{i}}(t) \exp \left(-j 2 \pi k \frac{t}{N_{c}}\right) \\
H_{u, m}(k)=\sum_{t=0}^{N_{c}-1} h_{u, m} \exp \left(-j 2 \pi k \frac{t}{N_{c}}\right) \\
H_{u_{i}, m}(k)=\sum_{t=0}^{N_{c}-1} h_{u_{i}, m} \exp \left(-j 2 \pi k \frac{t}{N_{c}}\right) \\
N_{m}(k)=\frac{1}{\sqrt{N_{c}}} \sum_{t=0}^{N_{c}-1} n_{m}(t) \exp \left(-j 2 \pi k \frac{t}{N_{c}}\right) .
\end{array}\right.
$$

The first term in (3) is the desired signal, the second term is the MUI, the third term is the CCI, and the last term is the noise component.

The received signals $\left\{R_{\mathrm{m}}(k) ; m=0 \sim N_{\mathrm{r}}\right\}$ are then expressed in a matrix form as

$$
\mathbf{R}(k)=\mathbf{H}_{0}(k) S_{0}(k)+\sum_{u=1}^{U-1} \mathbf{H}_{u}(k) S_{u}(k)+\sum_{i=1}^{B} \sum_{u_{i}=0}^{U_{i}-1} \mathbf{H}_{i, u_{i}}(k) S_{i, u_{i}}(k)+\mathbf{N}(k),
$$

where $\boldsymbol{R}(k)=\left[R_{0}(k), R_{1}(k) \cdots R_{N_{r}-1}(k)\right]^{T}, \quad H_{u}(k)=\left[\begin{array}{lllll}H_{u, 0}(k) & H_{u, 1}(k) & \cdots & H_{u, N_{r}-1}(k)\end{array}\right]^{T}$, and $N(k)=\left[\begin{array}{llll}N_{0}(k) & N_{1}(k) & \ldots & N_{N_{r}-1}(k)\end{array}\right]^{T}$ with $[\cdot]^{T}$ representing the transpose operation.

\section{SC-FDAAA}

The structure of the SC-FDAAA transceiver in both CAN system and DAN system can be generalized and shown in Fig. 3. Binary data sequence is modulated and divided into a sequence of blocks of $N_{c}$ data symbols. The last $N_{g}$ symbols in each block are copied and inserted as CP into the guard interval (GI) and placed at the beginning of each block. The received signal is transformed by an $N_{c}$-point fast Fourier transform (FFT) into the frequency domain signal and SC-FDAAA weight control is then performed on each frequency as 


$$
\widetilde{R}(k)=\boldsymbol{W}^{T}(k) \boldsymbol{R}(k)
$$

where

$$
\mathbf{W}(k)=\left[W_{0}(k), \mathrm{L}, W_{N_{r}-1}(k)\right]^{T} .
$$

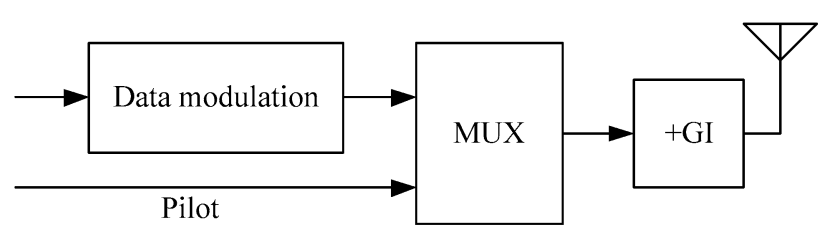

(a) Transmitter

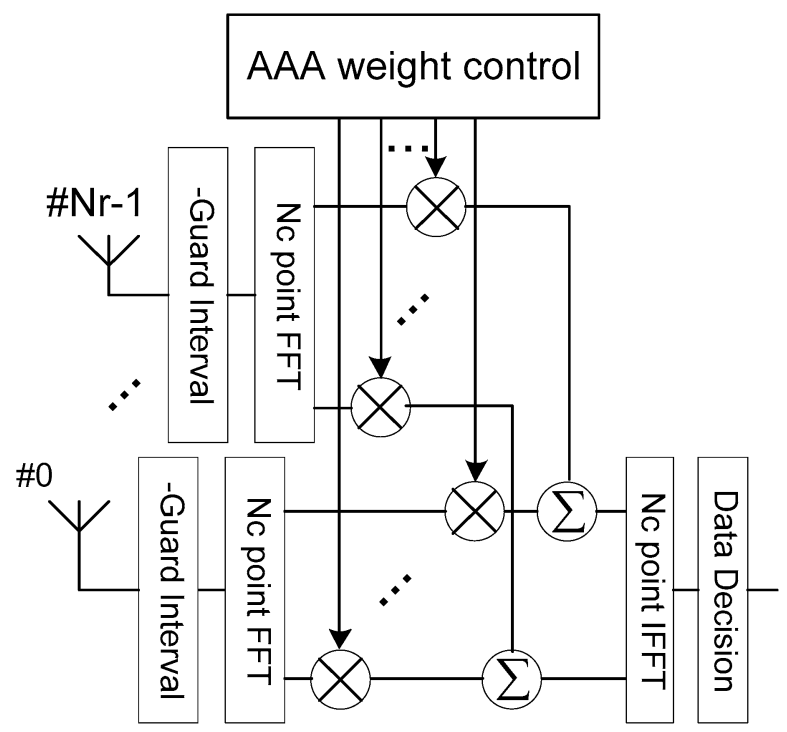

(b) Receiver

Figure 3. SC-FDAAA transceiver structure.

The SC-FDAAA weight that minimizes the mean squared error (MSE) between $\widetilde{R}(k)$ and the reference signal $S_{0}(k)$ (the pilot signal will be used as the reference signal) is given by [10, 11]

$$
\mathbf{W}(k)=\mathbf{C}_{r r}^{-1}(k) \mathbf{C}_{r d}(k),
$$


where $C_{r r}(k)=E\left\{\boldsymbol{R}^{*}(k) R(k)\right\}$ is the correlation matrix of the received signal and $C_{r d}(k)=E\left\{R^{*}(k) S_{0}(k)\right\}$ is the cross-correlation vector between the received signal and the reference signal, and * denotes complex conjugate operation.

$$
\begin{aligned}
\mathbf{C}_{r r}(k) & =E\left\{\mathbf{R}^{*}(k) \mathbf{R}(k)\right\} \\
& =\mathbf{A}_{0}^{*}(k) \mathbf{A}_{0}(k)+\sum_{u=1}^{U-1} \mathbf{A}_{u}^{*}(k) \mathbf{A}_{u}(k)+\sum_{i=1}^{B} \sum_{u_{i}=0}^{U_{i}-1} \mathbf{A}_{i, u_{i}}^{*}(k) \mathbf{A}_{i, u_{i}}(k)+N_{0} \mathbf{I} \\
& =\mathbf{A}_{0}^{*}(k) \mathbf{A}_{0}(k)+\mathbf{N}^{\prime}(k),
\end{aligned}
$$

and

$$
\mathbf{C}_{r d}(k)=E\left\{\mathbf{R}^{*}(k) S_{0}(k)\right\}=\mathbf{A}_{0}(k) S_{0}(k)
$$

where $A_{0}(k)=H_{0}(k) S_{0}(k)[12], N^{\prime}=\sum_{u=1}^{U-1} A_{u}^{*}(k) A_{u}(k)+\sum_{i=1}^{B} \sum_{u_{i}=0}^{u_{i}-1} A_{i, u_{i}}^{*}(k) A_{i, u_{i}}(k)+N_{0} \boldsymbol{I}$ is used to represent the interference plus noise.

In the next, time domain signal block estimate is then obtained by an $N_{c}$ - point IFFT for data decision as

$$
\hat{d}(t)=\frac{1}{\sqrt{N_{c}}} \sum_{k=0}^{N_{c}-1} \widetilde{R}(k) \exp \left(j 2 \pi k \frac{t}{N_{c}}\right) .
$$

\section{Post SC-FDAAA SINR}

The post SC-FDAAA SINR on the $k^{\text {th }}$ frequency can be evaluated by [13]

$$
\Gamma(k)=\frac{\mathbf{W}^{H}(k) \mathbf{R}_{s}(k) \mathbf{W}(k)}{\mathbf{W}^{H}(k) \mathbf{R}_{N^{\prime}}(k) \mathbf{W}(k)},
$$

where $\boldsymbol{R}_{s}(k)$ and $\boldsymbol{R}_{N}{ }^{\prime}(k)$ are the auto-correlation matrix of the received desired signal and the interference plus noise, respectively.

Property: if a matrix $\mathbf{Z}$ can be written as $\mathbf{Z}=\boldsymbol{T}^{-1}+\mathbf{P} \mathbf{Q}^{-1} \boldsymbol{P}^{*}$, then the inverse matrix of $\mathbf{Z}$ can be obtained by [14] 


$$
\mathbf{Z}^{-1}=\mathbf{T}-\mathbf{T P}\left(\mathbf{Q}+\mathbf{P}^{*} \mathbf{T P}\right)^{-1} \mathbf{P}^{*} \mathbf{T}
$$

Let $\boldsymbol{Z}=\boldsymbol{C}_{r r}(k), \boldsymbol{T}=\boldsymbol{R}_{N I}^{-1}(k), \boldsymbol{P}=\boldsymbol{A}_{0}^{*}(k)$ where $A_{0}(k)=\boldsymbol{H}_{0}(k) S_{0}(k)$ and $\boldsymbol{Q}=\boldsymbol{I}$, then the inverse matrix $C_{r r}^{-1}(k)$ can be calculated by submitting $Z, T, P$ and $I$ into

$$
\begin{aligned}
\mathbf{C}_{r r}^{-1} & =\mathbf{R}_{N^{\prime}}^{-1}(k)-\mathbf{R}_{N^{\prime}}^{-1}(k) \mathbf{A}_{0}^{*}(k)\left[\mathbf{I}+\mathbf{A}_{0}(k) \mathbf{R}_{N^{\prime}}^{-1} \mathbf{A}_{0}^{*}(k)\right]^{-1} \mathbf{A}_{0}(k) \mathbf{R}_{N^{\prime}}^{-1}(k) \\
& =\mathbf{R}_{N^{\prime}}^{-1}(k)\left[\mathbf{I}-\frac{\mathbf{A}_{0}^{*}(k) \mathbf{A}_{0}(k) \mathbf{R}_{N^{\prime}}^{-1}(k)}{\mathbf{I}+\mathbf{A}_{0}(k) \mathbf{R}_{N^{\prime}}^{-1} \mathbf{A}_{0}^{*}(k)}\right] \\
& =\left[\frac{1}{1+\mathbf{A}_{0}(k) \mathbf{R}_{N^{\prime}}^{-1} \mathbf{A}_{0}^{*}(k)}\right] \mathbf{R}_{N^{\prime}}^{-1}(k)
\end{aligned}
$$

The SC-FDAAA weight is then obtained by substituting (9) and (13) into (7), given by

$$
\mathbf{W}(k)=\left[\frac{1}{1+\mathbf{A}_{0}(k) \mathbf{R}_{N^{\prime}}^{-1} \mathbf{A}_{0}^{*}(k)}\right] \mathbf{R}_{N^{\prime}}^{-1}(k) \mathbf{A}_{0}(k) S_{0}(k) .
$$

Finally, the SINR after the weight control can be expressed, by substituting (14) into (9), as

$$
\Gamma(k)=\mathbf{A}_{0}(k) \mathbf{R}_{N^{\prime}}^{-1}(k) \mathbf{A}_{0}^{*}(k) .
$$

\section{Simulation results}

Performance of DAN SC-FDAAA system will be investigated in this section, as a reference for comparison, the performance of CAN SC-FDAAA will also be evaluated. Cellular structures using FRF =1, 3, 4, 7, 9 and 12 will be considered. The parameters used to generate the results are listed in Tab. I. No channel coding is used for simplicity, and we assume that the transmit signal to noise ratio (SNR) is $10 \mathrm{~dB}$. The distributed antennas are located in a cell as shown in Fig. 4. In this study, scheduling among the distributed antennas is not considered. The scheduling algorithm and more complicated situation remain as the topics of our future work.

BER performance is investigated at first. In order to find out behaviors of both average BER and outage BER, the cumulative distribution functions (C.D.Fs) of BER performance are calculated and shown in Figs. 5-8 where the FRF equals to 1, 3, 4 and 7, respectively. For the cases of FRF 9 and FRF 12, the results are similar to the case of FRF 7, therefore those results are not shown for brevity. Fig. 5 shows a comparison between the C.D.Fs of BER in DAN system 
and CAN system when $\mathrm{FRF}=1$. The $\mathrm{x}$ axis is the BER abscissa and $\mathrm{y}$ axis is the probability that BER<abscissa. It can be observed that DAN SC-FDAAA outperforms CAN SC-FDAAA by having better BER performance. It can also be observed that when the number of users increases, the BER performance of SC-FDAAA will degrade in both DAN and CAN systems, which can be intuitionally expected due to the reduction of degree of freedom. From the results shown in Figs. 6-8, it can be further observed that when FRF increases, the C.D.F. curves of BER performance "shift" right-side, which means that the BER performance improves due to the reduction of CCI power. In addition, DAN SC-FDAAA always achieves better BER performance than CAN SC-FDAAA no matter how FRF varies. The results of BER performance have shown that the distributed nature of DAN system can significantly improve the BER performance of SC-FDAAA over CAN system.

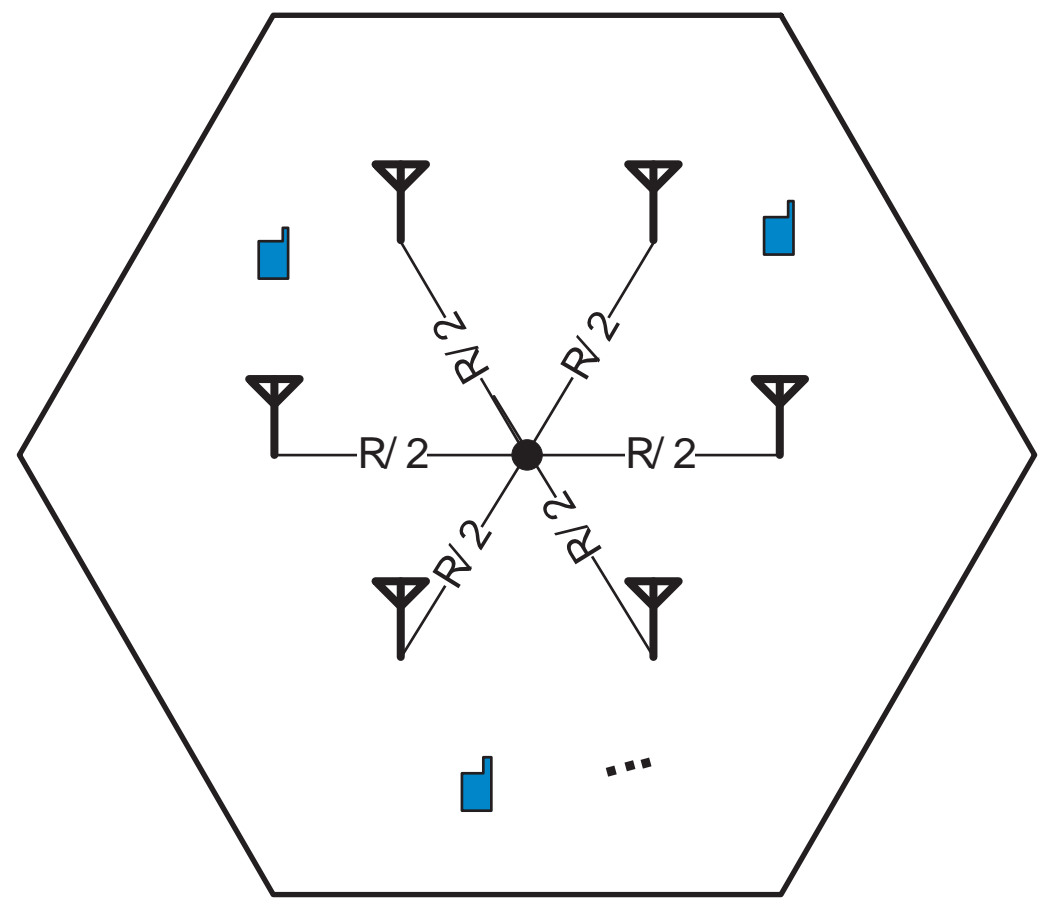

Figure 4. Antenna distribution in DAN system. 


\begin{tabular}{|c|c|c|}
\hline \multicolumn{2}{|r|}{ Modulation } & QPSK \\
\hline \multirow[t]{4}{*}{ Channel } & Channel Model & Frequency selective block Rayleigh fading \\
\hline & Number of paths & $L=16$ \\
\hline & Power delay profile & Uniform \\
\hline & Path loss & $a=3.5$ \\
\hline \multicolumn{2}{|r|}{ SNR } & $10 \mathrm{~dB}$ \\
\hline \multicolumn{2}{|r|}{ Number of co-channel cells } & $B=6$ \\
\hline \multicolumn{2}{|r|}{ Number of antennas of mobile user } & 1 \\
\hline \multicolumn{2}{|r|}{ Number of users per cell } & $U=2 \sim 6$ \\
\hline \multicolumn{2}{|r|}{ User location distribution } & Random \\
\hline \multicolumn{2}{|r|}{ Number of antennas } & $N_{r}=6$ \\
\hline \multicolumn{2}{|r|}{ FFT (IFFT) points } & $N_{c}=256$ \\
\hline
\end{tabular}

Table 1. Simulation Parameter

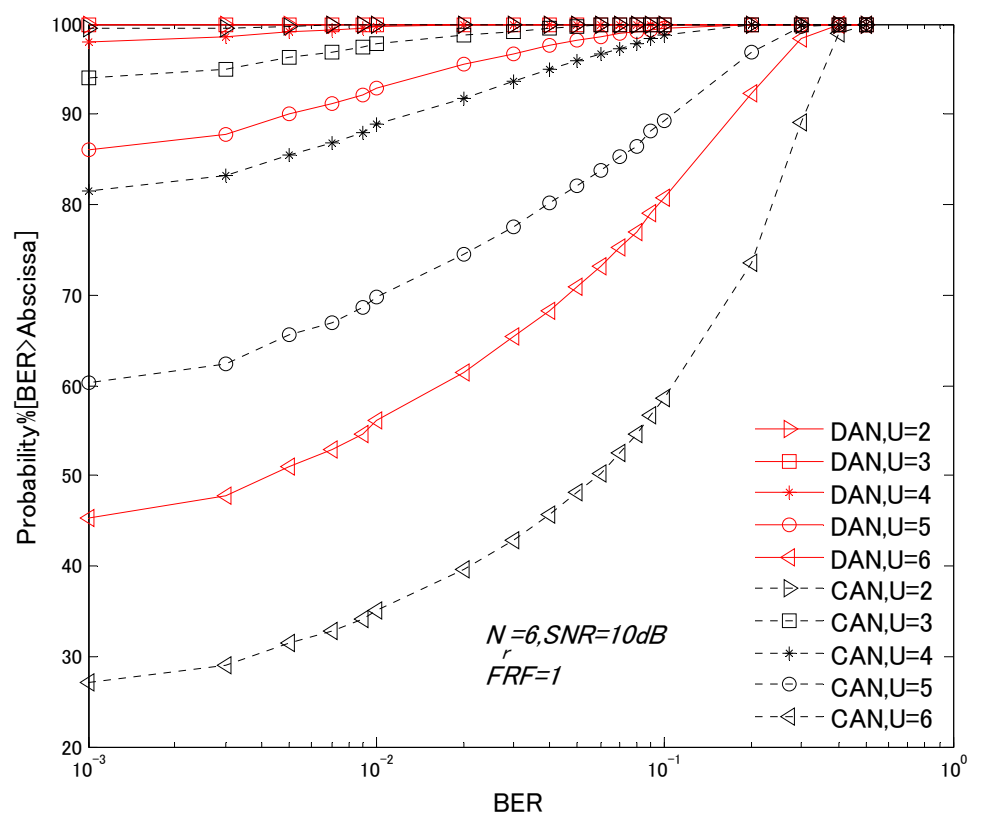

Figure 5. C.D.F. of BER performance, $F R F=1$. 


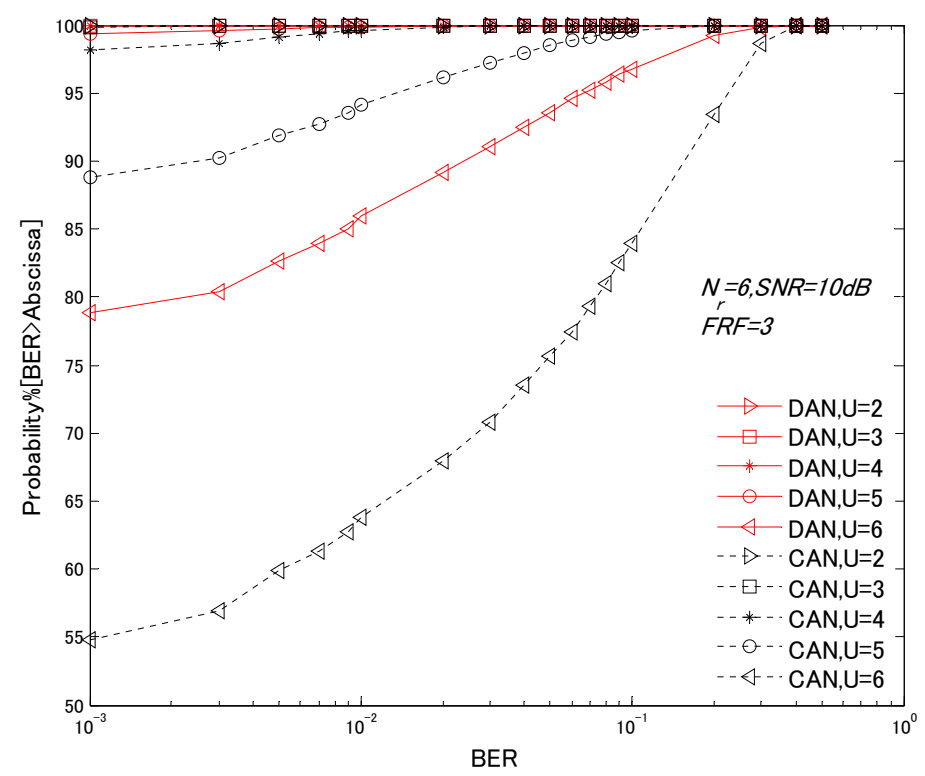

Figure 6. C.D.F. of BER performance, $F R F=3$.

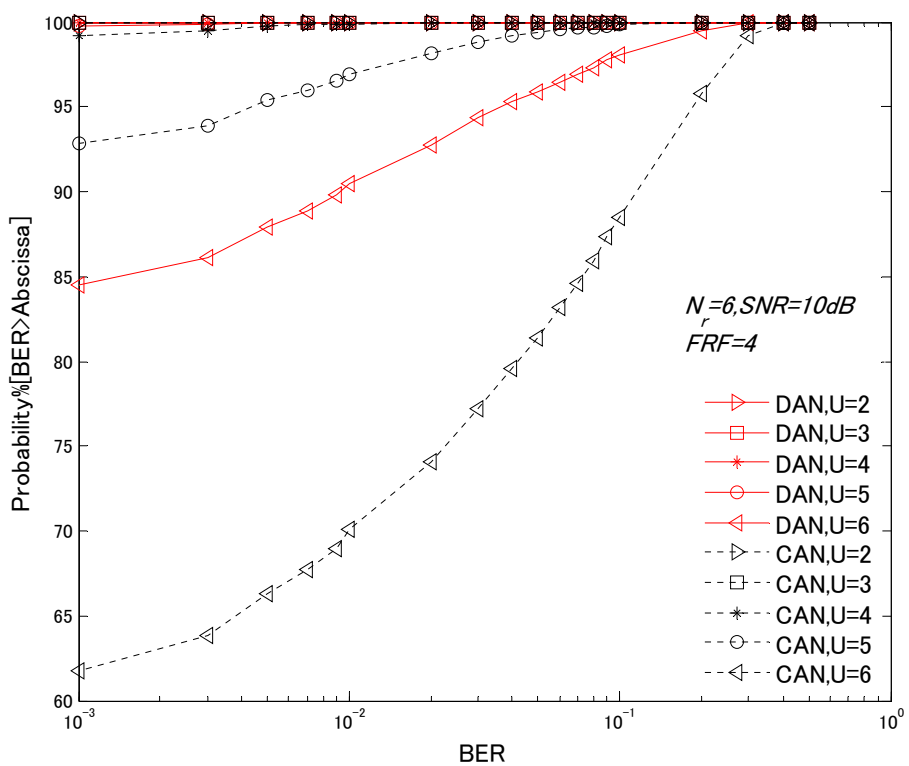

Figure 7. C.D.F. of BER performance, $F R F=4$. 


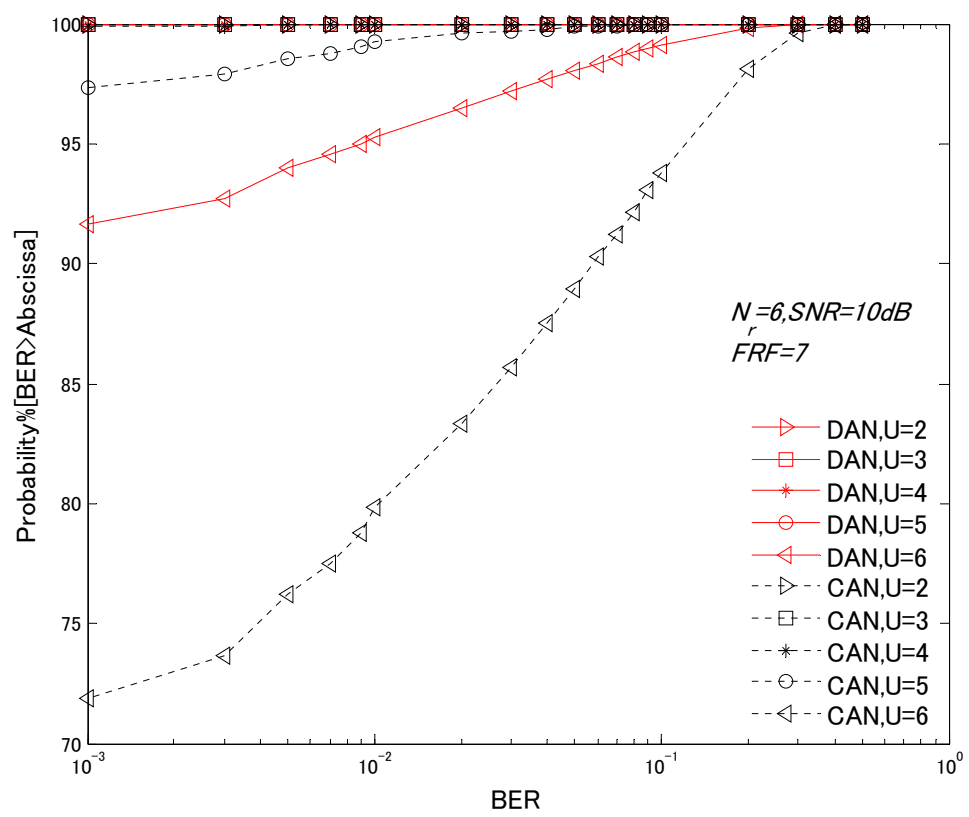

Figure 8. C.D.F. of BER performance, $F R F=7$.

System capacity given by bits/s/Hz can be calculated by Shannon capacity definition [15-16] using the SINR given in (15). However, the number of users that can be accommodated is a practical criterion to be considered as system capacity. Therefore, the following results will focus on the number of simultaneous users instead of the value given by bits/s/Hz. The average BER performance of DAN SC-FDAAA as a function of FRF is shown in Fig. 9. Since un-coded system is assumed, average $\mathrm{BER}=10^{-2}$ is used as a criterion to see how many users can be accommodated by using DAN SC-FDAAA. In the next, link capacity (maximum number of users/cell) and cellular link capacity (link capacity/FRF) of DAN SC-FDAAA are evaluated and the results are shown in Fig. 10 and Fig. 11.

Fig. 10 shows the link capacity of DAN SC-FDAAA and CAN SC-FDAAA. It is shown that 4 users can be accommodated by DAN SC-FDAAA when FRF 1 is used while 2 users can be accommodated by CAN SC-FDAAA, therefore, the link capacity can be doubled by using DAN SC-FDAAA. As the FRF increases, link capacities increase as the CCI power decreases. And when FRF is larger than 4,6 users can be accommodated. Since $N_{r}=6$ and the AAA receiver can deal with up to $N_{r}-1$ interference, it can be concluded that the maximum number of users/ cell of the DAN SC-FDAAA can approach its maximum value when FRF is larger than 4. 


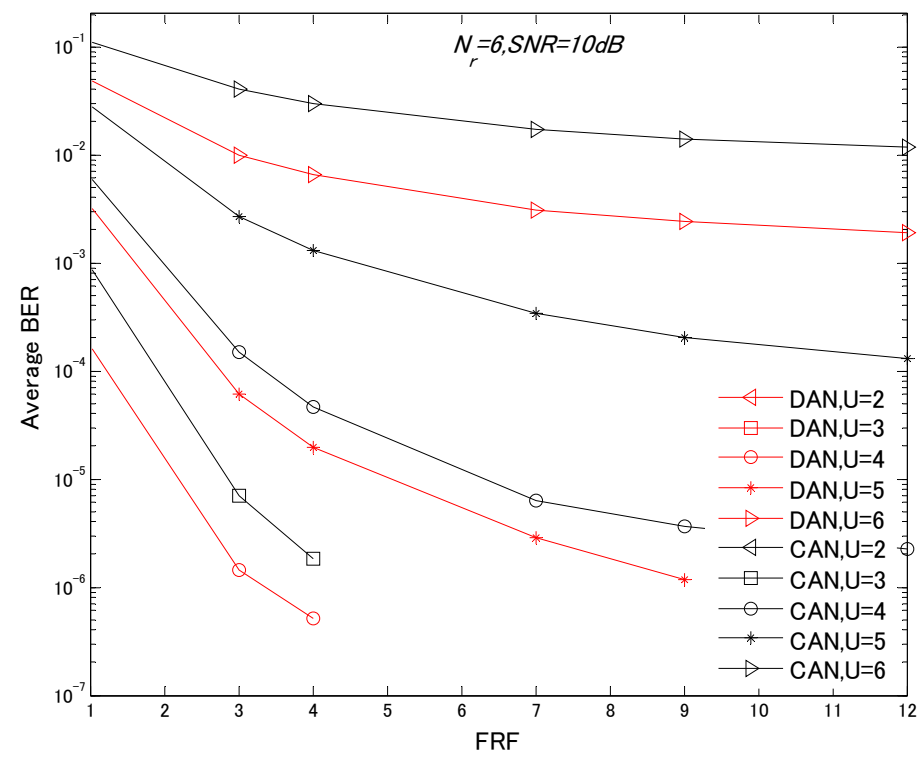

Figure 9. Average BER.

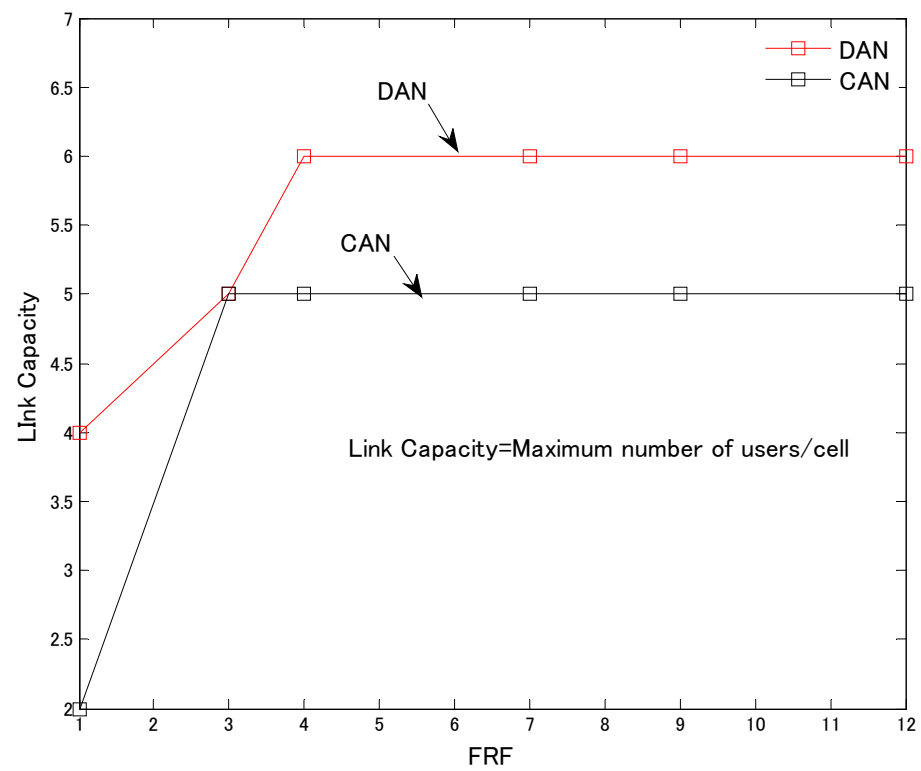

Figure 10. Link capacity. 


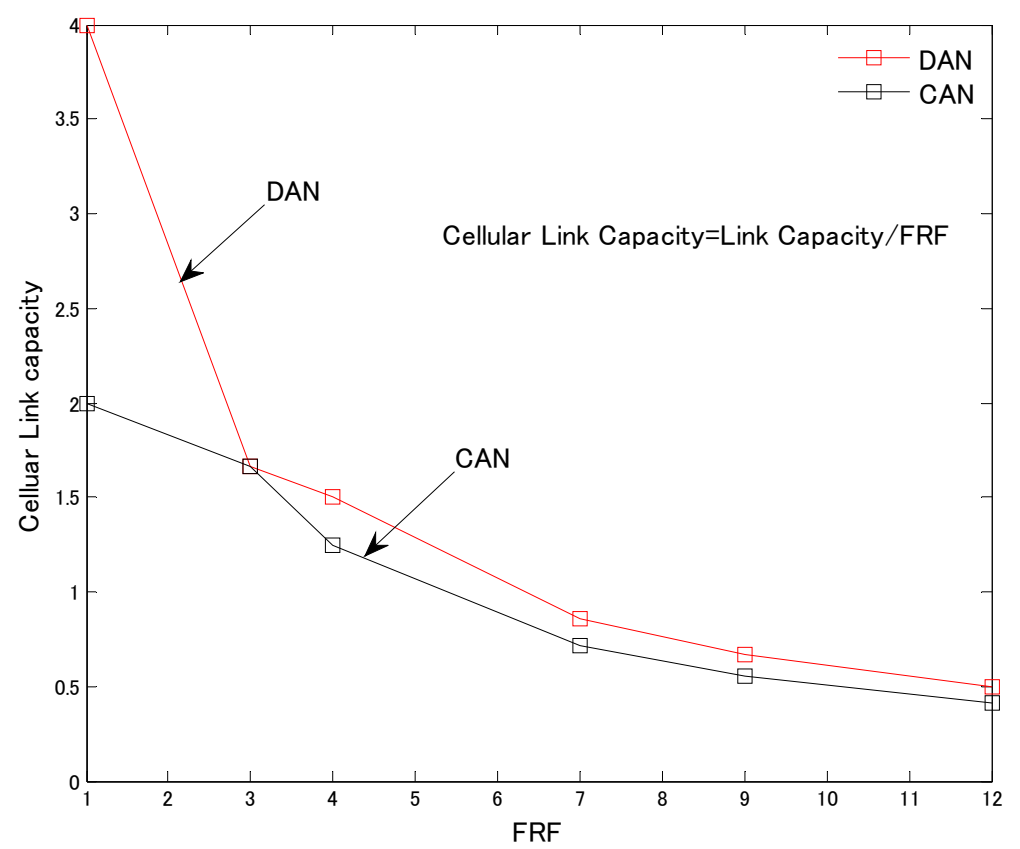

Figure 11. Cellular link capacity.

Larger FRF means more bandwidth will be consumed. In order to measure the spectrum efficiency, cellular link capacity is calculated and the results are shown in Fig. 11. It is shown that cellular link capacity of DAN SC-FDAAA can achieve its' maximum value when FRF =1 and decreases when FRF increases. Note that in our previous work on the cellular link capacity for conventional cellular system [17], it has been pointed out that the cellular link capacity can be maximized by using FRF $=1$ in the area near cell center and FRF $=3$ in the area near the cell edge. Therefore, by using the DAN SC-FDAAA, a smaller FRF can be used and the spectrum efficiency can be greatly improved as a result. In addition, taking FRF $=1$ as an example, DAN SC-FDAAA achieves twice of the cellular link capacity as CAN SC-FDAAA does.

\section{Conclusions}

In this article, we presented the performance of DAN SC-FDAAA and also the performance of CAN SC-FDAAA as a reference. Both the BER distribution and capacity (link capacity and cellular link capacity) are given. The cellular link capacity can be maximized when FRF=1 by DAN SC-FDAAA. Therefore, the DAN SC-FDAAA can use the single frequency reuse (i.e., $F R F=1$ ) and improve the spectrum efficiency greatly. In addition, the performance of DAN SCFDAAA can benefit from the distributed nature of DAN, and it can double the cellular link capacity by using the single frequency reuse when compared with CAN SC-FDAAA. 


\section{Author details}

Wei Peng and Fumiyuki Adachi

*Address all correspondence to: peng@mobile.ecei.tohoku.ac.jp

Tohoku University, Japan

\section{References}

[1] J. G. Proakis, Digital Communications, fourth edition, New York: McGraw Hill, 2001.

[2] R. Price and P. E. Green, "A Communication Technique for Multipath Channels," Proc. IRE, vol. 46, pp. 555-570, March 1958.

[3] D. Falconer, S. L. Ariyavistakul, A. Benyamin-Seeyar and B. Edison, "Frequency Domain Equalization for Single-carrier Broadband Wireless Systems," IEEE Communication Magazine, vol. 40, pp. 58-66, April 2002.

[4] R. Van Nee and R. Prasad, OFDM for Wireless Multimedia Communications, Arctech House Publishers, UK, 1999.

[5] F. Adachi, K. Takeda, T. Obara, T. Yamamoto and H. Matsuda, "Recent advances in single-carrier frequency-domain equalization and distributed antenna network," IEEE ICICS 2009, pp.1-5, March 2009.

[6] K. Sivanesan and N. C. Beaulieu, "Outage and BER of MRC Diversity in Band-limited Micro-cellular Systems with CCI," IEEE Communications Letters, vol. 9, Issue. 3, pp. 207-209, March 2005.

[7] J. Zhang and J. Andrews, J, "Distributed Antenna Systems with Randomness", IEEE Transactions on Wireless Communications, vol. 7, no. 9, pp. 3636 - 3646,Sept. 2008.

[8] W. Peng and F. Adachi, "Frequency Domain Adaptive Antenna Array for Broadband Single-Carrier Uplink Transmission," IEICE Transactions on Communications, vol. E94-B, no. 7, pp. 2003-2012, July. 2011.

[9] W. Peng and F. Adachi, "Single-Carrier Frequency Domain Adaptive Antenna Array for Distributed Antenna Network," IEEE ICCS, pp. 1-5, Dec. 2010.

[10] Ahmed EI Zooghby, Smart Antenn Engineering, Arctech House Publisher, 2005.

[11] Simon Haykin, Adaptive Filter Theory, New York: Prentice Hall, 2002.

[12] J. H. Winters, "Signal Acquisition and Tracking with Adaptive Arrays in the Digital Mobile Radio System IS-36 with Flat Fading," IEEE Trans. Vehicular Technology, vol. 42, pp. 377-384, Nov. 1993. 
[13] K. Hamdi, "On the Statistics of Signal-to-interference plus Noise Ratio in Wireless Communications," IEEE Transactions on Communications, vol. 57, no. 11, pp. 3199 3204, Nov. 2009.

[14] M. A. Woodbury, “Inverting Modified Matrices,” New Jersey: Princeton, 1950.

[15] C.E. Shannon, "A Mathematical Theory of Communication I," Bell System Technical Journal, pp. 379-423, July 1948.

[16] C.E. Shannon, "A Mathematical Theory of Communication II," Bell System Technical Journal, pp. 623-656, October, 1948.

[17] W. Peng and F. Adachi, "Hybrid Frequency Reuse Scheme for Cellular MIMO Systems," IEICE Trans. Communications, vol. E92-B, pp. 1641-1648, May 2009. 